\title{
Investigation of reattachment length for a turbulent flow over a backward facing step for different step angle
}

\author{
Ajay Pratap Singh, Akshoy Ranjan Paul ${ }^{*}$, Pritanshu Ranjan \\ Department of Applied Mechanics, Motilal Nehru National Institute of Technology, Allahabad, INDIA \\ *Corresponding Author: e-mail: arpaul2k@yahoo.co.in, Tel +91-532-2271212, Fax.+91-532-2271200
}

\begin{abstract}
Computational investigations are carried out on flow through a channel having a single backward facing step. The effect of step angle and expansion ratio on separation length is studied and compared with experimental results reported in literature. The step angles considered are $15^{\circ}, 30^{\circ}, 45^{\circ}$ and $90^{\circ}$, whereas the expansion ratios considered are 1.48 and 2.0 . A two-dimensional (2D), segregated, renormalized group (RNG) $k-\varepsilon$ turbulence model has been chosen for CFD simulations. The analysis of the results showed that with the increase in step angle and expansion ratio, the reattachment length increases. Effect of Reynolds number is also studied by varying it over a range 15000 to 64000 and it also shows that the reattachment length increases with an increase of the Reynolds number.
\end{abstract}

Keywords: Backward facing step, flow separation, reattachment length, computational fluid dynamics (CFD), turbulence model.

\section{Introduction}

Flow with separation and reattachment has long been a subject of fundamental fluid dynamics research. The presence of a separated flow, together with a reattaching flow, gives rise to increased unsteadiness, pressure fluctuations, structure vibrations and noise, as it also exhibits an unsteady structure with a large scale vortex in the separated shear layer and low-frequency motion around the reattachment region with fluctuation of an instantaneous reattachment point (Troutt et al., 1984). The flow over a single side backward facing step provides a classic example of above said flow field. The simple and easily attainable two-dimensional (2D) geometry makes any step-geometry a popular choice to study separation phenomenon. Furuichi et al. (2004) studied a twodimensional backward facing step and measured the velocity fluctuations by using a multi-point LDV and correlate the moving path of vortex shedding and velocity fluctuation.

But in past, only $90^{\circ}$ step has often been investigated, the flow over the inclined step attracted less attention. Ruck and Makiola (1989) performed experiments on inclined steps for different Reynolds number and expansion ratio and studied its effect on the reattachment length. Their study concluded that by increasing the area ratio or inclination angle reattachment length increases for a particular Reynolds number. In this study, computational analysis is carried out to solve a flow field in a two-dimensional backward facing step and to find the effect of step inclination on the reattachment length of the separated flow. Fig. 1 shows the schematic diagram of the problem.

This paper was presented at the $5^{\text {th }}$ National Conference on Applicable Mathematics in Wave Mechanics and Vibrations (WMVC2010) held at Kakatiya University, Warangal, India, 13-15 March, 2010. 


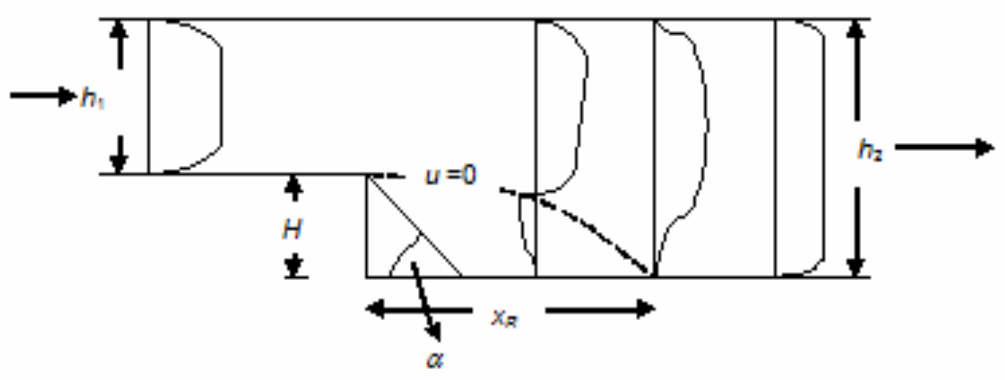

Fig. 1. Backward facing step with different inclination angle

\section{Computational Techniques}

For the present study, all the cases considered are summarized in the table 1. The modelling is done in 'Gambit' modelling tool for the aforementioned geometries. Pure quadrilateral meshing is used to get structured mesh with total number of 130000 cells and the same is shown in Fig.2.

Table 1: Configurations Examined in present study

\begin{tabular}{ccccccc}
\hline \multirow{2}{*}{$\operatorname{ER}\left(\boldsymbol{h}_{\mathbf{2}} / \boldsymbol{h}_{\mathbf{1}}\right)$} & $\mathbf{R e}$ & $\boldsymbol{u}(\mathbf{m} / \mathbf{s})$ & \multicolumn{4}{c}{ Step angle $\left(\boldsymbol{\alpha}^{\circ}\right)$} \\
\hline \multirow{3}{*}{1.48} & 15000 & 2.20 & 15 & 30 & 45 & 90 \\
\cline { 2 - 7 } & 47000 & 6.87 & 15 & 30 & 45 & 90 \\
\cline { 2 - 7 } & 64000 & 10.0 & 15 & 30 & 45 & 90 \\
\hline \multirow{3}{*}{2.0} & 15000 & 2.20 & 15 & 30 & 45 & 90 \\
\cline { 2 - 7 } & 47000 & 6.87 & 15 & 30 & 45 & 90 \\
\cline { 2 - 7 } & 64000 & 10.0 & 15 & 30 & 45 & 90 \\
\hline
\end{tabular}

RNG $k-\varepsilon$ model is used to predict the flow field Flow past the step involves recirculation (swirl) and the effect of swirl on turbulence is included in the RNG model, due to which accuracy of the model further increases.

A steady state based implicit solver is used to achieve convergence. Second-order upwind scheme was used for the discretization of all the equations to achieve higher accuracy in results. Velocity-pressure coupling is established by pressure-velocity correlation using a SIMPLE algorithm. Under-relaxation factors are used for all equation to satisfy Scarborough condition (Fluent's user guide, 2006). Residuals are continuously monitored for continuity, $x$-velocity, $y$-velocity, $z$-velocity, $k$, and $\varepsilon$. Convergence of the solution is assumed when the values of all residuals goes below $10^{-6}$. Standard wall function is used to solve for the near wall treatment, as $y^{+}$is more than 30 in the whole domain.

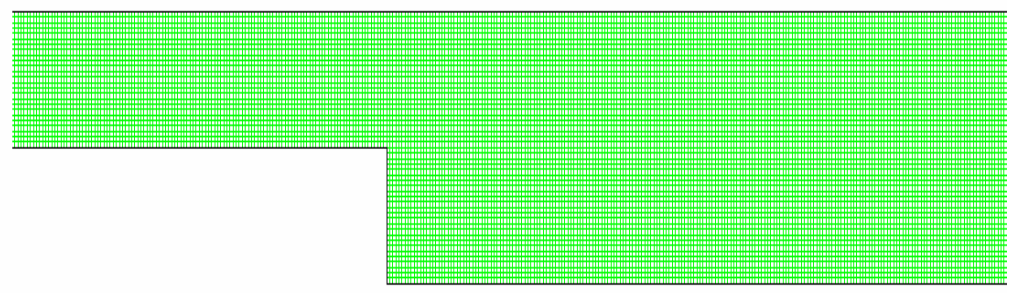

Fig. 2. Backward step with quadrilateral meshing.

\section{Results and Discussion}

The commercially available code 'Fluent 6.3' is validated using the experimental data reported by Ruck and Makiola (1989). Fig. 3 represents the variation of non-dimensional attachment length with Reynolds number for experimental and computational cases. It can be observed that the computational result closely follows the experimental trend. 
It can be seen from Fig. 3 that for a particular step angle at low expansion ratio (ER=1.48), the reattachment length doesn't increase steeply with the increase in Reynolds number. At $90^{\circ}$ step angle, a maximum of $4.28 \%$ increment in the reattachment length can be observed for Reynolds number 64000 as compared to that with 15000 .

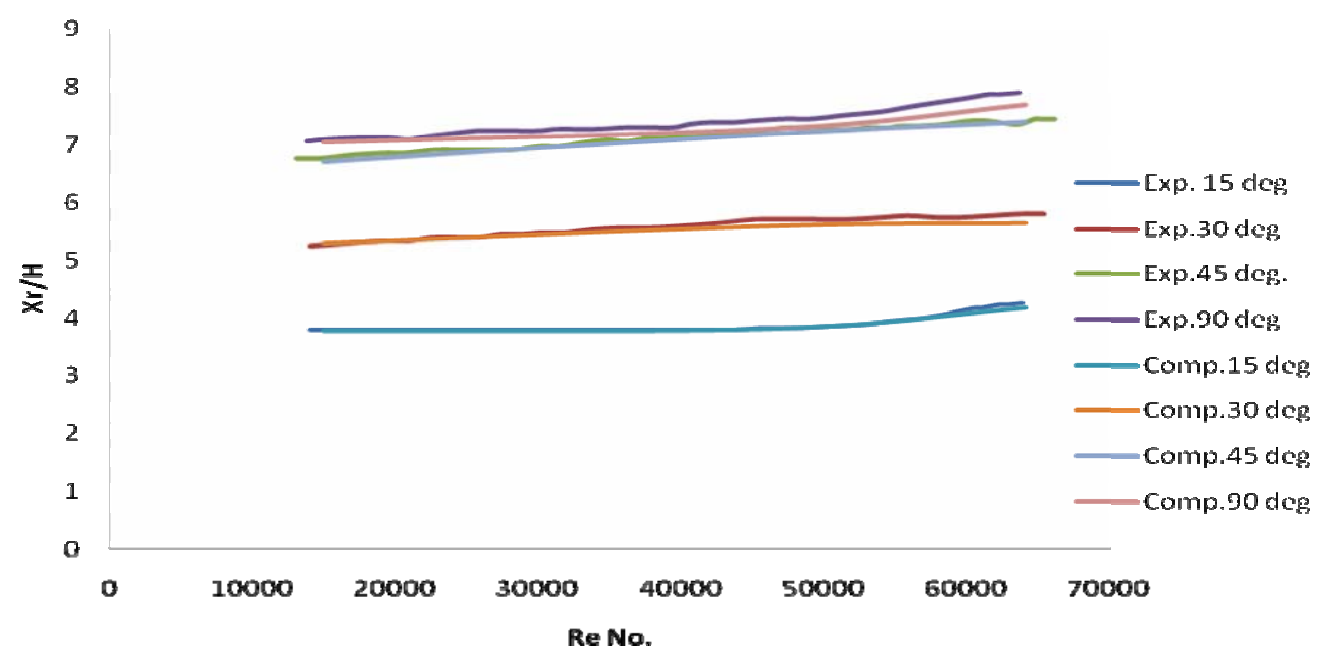

Fig. 3. Variation of $X_{\mathrm{r}} / h$ with Reynolds number for different step angles, ER $=1.48$.

The variation of reattachment length with step angle for constant Reynolds number for ER=2 is shown in Fig. 4. It has been observed that as the step angle increases, the reattachment length increases initially till $45^{\circ}$ and remains almost constant thereafter, this fact can also be attributed from Fig. 3 where the lines for reattachment length for $45^{\circ}$ and $90^{\circ}$ almost coincides even at $\mathrm{ER}=1.48$. For $\mathrm{ER}=2$, Fig. 4 shows that for a particular step angle, the reattachment length increases appreciably with increases in Reynolds number.

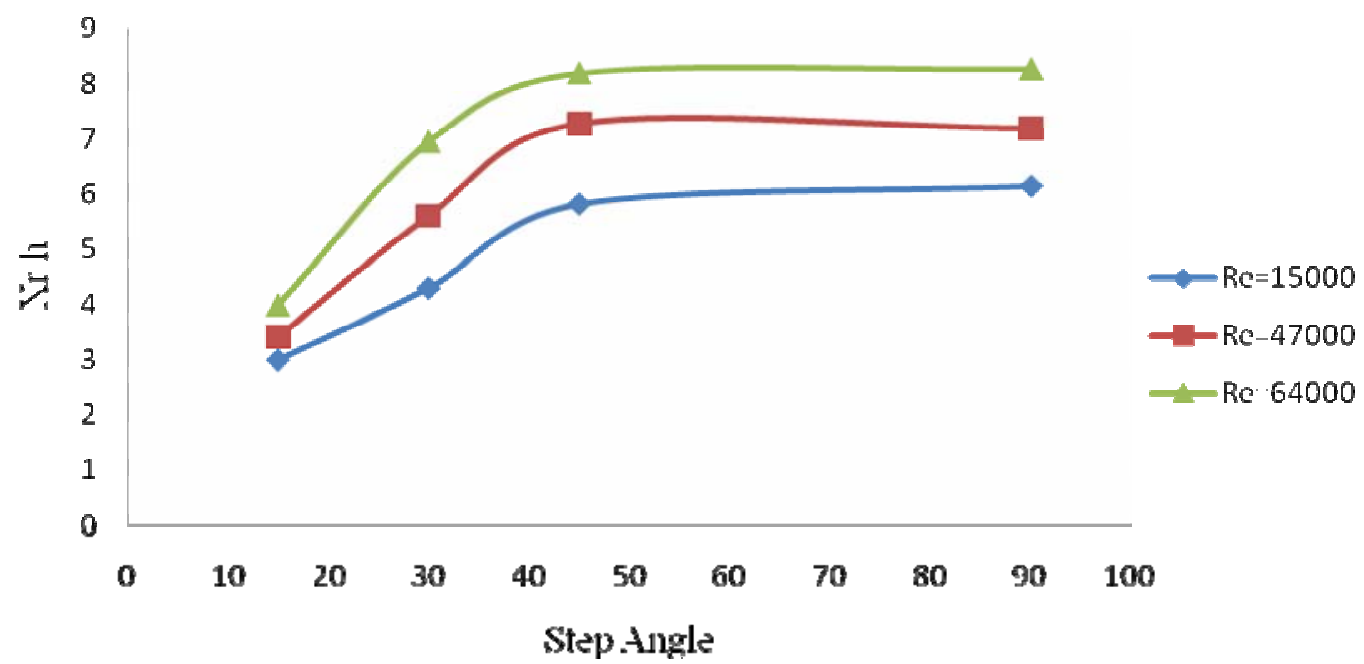

Fig. 4. Variation of reattachment length with step angle, for ER = 2.0.

Unlike for $90^{\circ}$ step angle at low expansion ratio (1.48), the value of reattachment length increases by a factor of 2 for $\mathrm{Re}=64000$ as compared to that for $\mathrm{Re}=15000$. This shows that as the expansion ratio is increased, the effect of Reynolds number also increases on reattachment length. Thus, for low expansion ratios, $X r$ is independent of Reynolds number. Further from Figs. 3 and 4, it can be well observed that for any expansion ratio, initially $X r$ value increases with step angle but becomes independent of it at higher values.

Further Fig. 5 shows the variation of $X r$ with the expansion ratio at two different Reynolds number. It was observed that for low Reynolds number (15000) the value of $X r$ decreases by $16.67 \%$ when expansion ratio is increased from 1.48 to 2 . This could be possible because at low Re and high ER, the flow gets enough space and time to reattach to the boundary, thereby reducing the reattachment length. But in case of higher Reynolds number $(\mathrm{Re}=64000)$, the reverse phenomena happens, i.e. the value of $X r$ increases with an increase of ER from 1.48 to 2. 


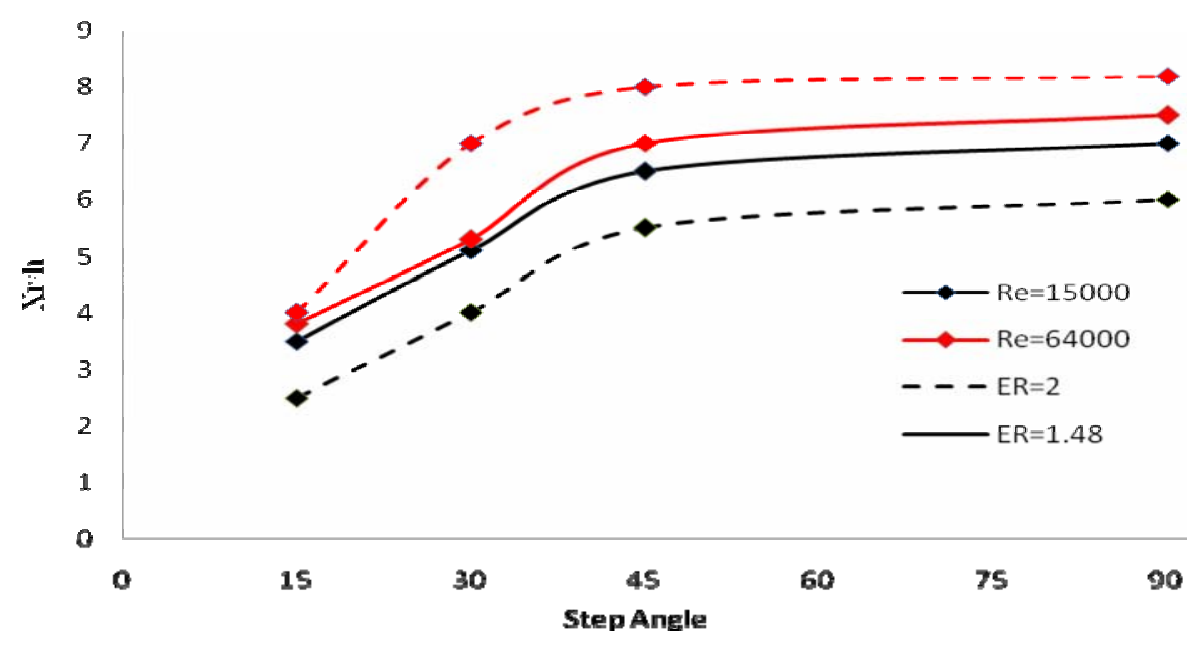

Fig. 5. Variation of reattachment length wihexpansion ratio at different Re.

The flow can be better visualized in form of streamline as shown in Fig. 6. It can be seen that there is a distinct secondary flow in the separated region near the corner.

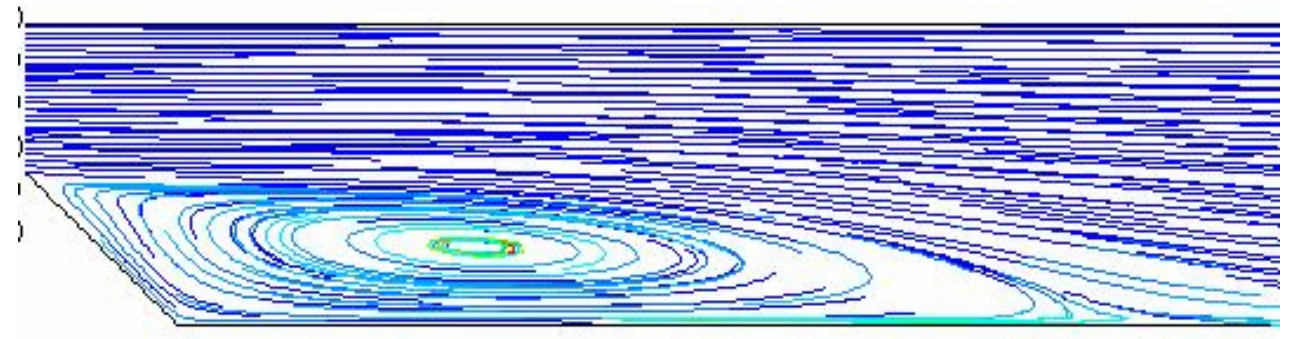

Fig. 6. Streamlines depicting secondary flow (enlarged view).

\section{Conclusions}

The flow characteristics over a two-dimensional backward facing step have been computationally simulated. The analysis of the results is summarized below.

- The non-dimensional reattachment length $\left(X_{r} / H\right)$ increases from 3.78 for a $15^{\circ}$ step angle to 7.1 for a $90^{\circ}$ step, at $\operatorname{Re}=$ 15000.

- The non-dimensionalized reattachment length also increases from 7.0 to 7.7 as Re increases from 15000 to 64000 for a $90^{\circ}$ step.

- With increase in expansion ratio from 1.48 to 2.0, the reattachment length decreases for low Reynolds number $(\mathrm{Re}=$ 15000) but increases for higher Reynolds number.

- For a particular Reynolds number, the reattachment length increases initially till $45^{\circ}$ and thereafter, remains almost constant.

\section{Nomenclature}

$\begin{array}{ll}\text { ER } & \text { expansion ratio, ER }=h_{2} / h_{1}, \text { dimensionless } \\ h_{1} & \text { inlet width, mm } \\ h_{2} & \text { outlet width, mm } \\ H & \text { step height, mm } \\ \mathrm{Re} & \text { Reynolds number, dimensionless } \\ u & \text { freestream velocity of air, mm } \\ u_{*} & \text { friction velocity at the nearest wall, } u_{*}=\sqrt{\tau_{w} / \rho}, \mathrm{m} / \mathrm{s} \\ X_{r} & \text { reattachment length, mm } \\ y & \text { distance to the nearest wall (normal to flow direction), mm }\end{array}$




$\begin{array}{ll}y^{+} & \text {dimensionless wall distance, } y^{+}=u_{*} y / v \\ \alpha & \text { step angle, degree } \\ v & \text { kinematic viscosity of fluid, } \mathrm{m}^{2} / \mathrm{s} \\ \rho & \text { density of fluid, } \mathrm{kg} / \mathrm{m}^{3} \\ \tau_{w} & \text { wall shear stress, } \mathrm{N} / \mathrm{m}^{2}\end{array}$

\section{Acknowledgement}

The authors thanks to the Department of Science \& Technology, Govt. of India for financing through DST-FIST grant to carry out this research.

\section{References}

Furuichi, N., Hachiga, T. and Kumada, M., 2004. An experimental investigation of a large-scale structure of a two-dimensional backward-facing step by using advanced multi-point LDV, Experiments in Fluids, Vol. 36, pp. 274-281.

Fluent User’s Guide, CFD software package, 2005. Ver. 6.3, Fluent, Lebanon, NH.

Ruck, B. and Makiola, B., 1989. Flow Separation over the inclined plane, In: K.Gerstan (Eds.), Numerical Fluid Mechanics (Vol. 40), Physics of Separated Flows: Numerical, Experimental, and Theoretical Aspects, Vieweg, Berlin.

Troutt, T. R., Scheelke, B. and Norman, T. R., 1984. Organized Structures in a Reattaching Separated Flow Field, J. Fluid Mech. Vol. 143, pp. 413-427.

\section{Biographical notes}

Ajay Pratap Singh has recently completed his M.Tech. in Fluids Engineering from Applied Mechanics department at Motilal Nehru National Institute of Technology, Allahabad, India. He is currently working as Assistant Professor in the Mechanical Engineering department at A.K. Garg Institute of Technology, Ghaziabad, India. He has published a couple of research papers in national and international conferences.

Akshoy Ranjan Paul is an Assistant Professor in the Department of Applied Mechanics, Motilal Nehru National Institute of Technology, Allahabad, India. He has received M. E. degree from Jadavpur University , Kolkata, India in 2002. He has more than nine years of experience in teaching and research. His current research area includes internal flow aerodynamics, flow control and turbulence modeling. He has published three textbooks and many technical papers in national and international conferences and journals. He visited a few universities in UK, Denmark, and Sweden for academic visit and technical training.

Pritanshu Ranjan is presently working as a visiting faculty member in Applied Mechanics department at Motilal Nehru National Institute of Technology, Allahabad, India. He did his M.Tech. in Fluids Engineering from the same department. He is involved in the flow control research group in the institute. He has published a few research papers in national and international conferences and journals.

Received September 2010

Accepted March 2011

Final acceptance in revised form March 2011 